\title{
Dental implant stability
}

Osseointegrated dental implants have been considered the most esthetical and functional alternative to missing teeth in the last decade. Regardless the recent trends and technology, the potential of implants failure is a significant concern for both of the dentist and the patient. Osseointegration is a patient-depended wound healing process affected by various factors.

\section{Successful osseointegration}

Successful osseointegration has been defined as a direct structural and functional connection existing between the living bone and the surface of the implant. The osseointegration mechanism is very similar to the primary bone healing. Thus, after surgical trauma, there is an inflammatory process, in which a mediator cascade promotes hematoma as well as circulatory alteration. Following, regeneration is developed and, consequently, the wound is replaced by bone tissue. Subsequently, wound maturation takes place by means of a remodeling mechanism, which is influenced by occlusion pressure. When an adequate regeneration occurs, there is a direct contact between the metal surface and bone tissue.

The term implant success used to describe ideal clinical conditions. It should include a time period of at least 12 months for implants serving as prosthetic abutments. The term early implant success is suggested for a span of 1 to 3 years, intermediate implant success for 3 to 7 years, and long-term success for more than 7 years. Criteria for implant success should serve as an aid to clinical follow-up and to help evaluate the clinical outcomes of different implant systems in research.

\section{Implant failure}

Implant failure (loss of osseointegration) should be distinction form the "implant complication". However, implant complication can lead to implant failure. The implant failure can be characterized by a connective tissue capsule that involves the implant. This capsule forms when the repair process occurs instead of regeneration. After injury, fibroblasts and vascular endothelial cells proliferate; a granulation tissue is formed. In the early failures, a repair process substitutes bone regeneration, resulting in a soft tissue capsule that surrounds the implant and promotes its mobility.

Success or failure of dental implants is multifactorial in origin. Factors related to site of insertion of dental implants, patients related factors, and the surgeon related factors are interacting together leading to success or failure of the dental implant. The implant failure can be classified as Early failure (the osseointegration is not established) and late failure (breakdown of the established osseointegration). There are various causes related to early failure (overheating, contamination and trauma during surgery, poor bone quantity and/or quality, lack of primary stability, and incorrect immediate load indication), and late failure (peri implantitis, occlusal trauma, and overloading).

\section{Patient factors}

Patient factors are important determinants of implant failure Patient local factors includes Oral hygiene, Gingivitis, Bone quantity/ quality, Adjacent infection/inflammation, Presence of natural teeth,
Volume 4 Issue 3 - 2016

\author{
Ayman F Hegab \\ Department of Oral \& Maxillofacial Surgery,Al-Azhar University, \\ Egypt \\ Correspondence: Ayman Hegab, Clinical Associate Professor \\ of Oral \& Maxillofacial Surgery,Al-Azhar University, Cairo, Egypt, \\ Tel +974333 I0I24, 0020100I98III2, \\ Email prof.aymanhegab@yahoo.com
}

Received: January 30, 2015 | Published: February 22, 2016

Periodontal status of natural teeth, Impaction of foreign bodies (including debris from surgical procedure) in the implant pocket, Soft tissue viability. Patient systemic factors includes Vascular integrity, Smoking, Alcoholism, Predisposition to infection, e.g. age, obesity, steroid therapy, malnutrition, metabolic disease (diabetes), Chemotherapy/radiotherapy, Hypersensitivity to implant components.

\section{Surgical factors}

Surgical factors includes Surgical trauma, Overheating, Perioperative bacterial contamination, (e.g. via saliva, perioral, skin, instruments, gloves). Lack of primary stability, surgical trauma, perioperative contamination and occlusal overload seem to be the most important causes of implant failure.

\section{Primary stability}

Primary stability of the dental implants mostly comes from the mechanical engagement with the cortical bone, while the secondary stability represents the biological stability through the bone regeneration and remodeling from the cancellous bone leading to osseointegration. The primary stability is considered as significant factor for the secondary stability. Primary stability is depending on the bone quantity \& quality (patient factor), surgical technique (surgeon factor), and the implant geometer and characteristics (implant factor). While secondary stability is depending on the primary stability, bone remodeling and modeling, and implant surface condition.

\section{Evaluation of the implant stability}

\section{Radiographic evaluation}

Radiographic evaluation is a simple non-invasive technique but it's associated with many problems. Crestal bone loss can be expected to be $1.5 \mathrm{~mm}$ in the first year, with $0.1 \mathrm{~mm}$ of subsequent annual bone loss. Radiographic evaluation of $0.1 \mathrm{~mm}$ bone loss is unrealistic and change of the crestal bone level cannot exactly predict the implant stability and failure. Moreover; evaluation of the crestal bone loss need standardized radiographs with temples for reliable and repeated measurements. Evaluation of the bone quality by plain X-ray cannot be detected until $40 \%$ of demineralization occurred. 


\section{Cutting torque resistance analysis (CTRA)}

CTRA is another method for assessment of implant stability intraoperative. CTRA measured the required current-fed electric motor in cutting off a unit volume of bone during implant insertion. This energy is significantly correlated with the with the bone density and subsequently with the implant stability. CTRA used intraoperative for identification of low-density bone. The technique consisted of incorporated torque gauge within the drilling unit to measure implant insertion torque in $\mathrm{Ncm}$ to indirectly represent $\mathrm{J} / \mathrm{mm} 3$. The main problem of CTRA is it doesn't give any information about bone quality until the osteotomy site prepared.

\section{Reverse torque test}

These measures the critical torque threshold required to destroy the bone-implant contact. Removal torque value (RTV) which indirect measurement of the critical torque threshold ranged from $45-48 \mathrm{Ncm}$ in Osseointegrated dental implants and $20 \mathrm{Ncm}$ is considered as acceptable criterion for successful osseointegration. While Branemark cautioned about the risk of implant failure due to irreversible plastic deformation of the peri-implant bone due to unnecessary loading with RTV of $20 \mathrm{Ncm}$ to the implant undergoing osseointegration.

\section{Percussion test}

It is one of the simplest methods for evaluation of the osseointegration. A clear ringing crystal sound indicates successful osseointegration while dull sound indicates no osseointegration.

\section{Periotest}

It is another non-invasive method to measure implant stability using electromagnetic driven and electronically tapping metallic rod in a hand piece. Contact time between the implant and tapping rod is measured on the time axis as a signal for analysis which converted to unique value called Periotest value (PTV)

\section{Resonance frequency analysis (RFA)}

It is diagnostic method which can measure of implant stability and bone density at various time points using vibration and a principle of structural analysis. Implant Stability Quotient (ISQ) created by Osstell to replace the old measurement unit (Hertz) of implant stability. Resonance frequency values ranging from 3500 to $8500 \mathrm{~Hz}$ are translated into 0 to $100 \mathrm{ISQ}$. A high value indicates the greater stability while low value refers to instability. The implant and bone act as a single unit after successful osseointegration, so, change of stiffness considered as change of Osseointegration of the dental implant. Factors influence the RFA includes; implant length, implant diameter, implant geometry, implant surface characteristics, abutment length, bone quality and quantity.

\section{Funding}

None.

\section{Acknowledgements}

None.

\section{Conflicts of interest}

Author declare that there is no conflict of interest. 